\title{
Estimation model of winter wheat disease based on meteorological factors and spectral information
}

\author{
Weiguo Li*, Yang Liu, Hua Chen and Cheng Cheng Zhang
}

\begin{abstract}
Wheat scab (WS, Fusarium head blight), one of the most severe diseases of winter wheat in Yangtze-Huaihe river region, whose monitoring and timely forecasting at large scale would help to optimize pesticide spraying and achieve the purpose of reducing yield loss. In the present study, remote sensing monitoring on WS was conducted in 4 counties in Yangtze-Huaihe river region. Sensitive factors of WS were selected to establish the remote sensing estimation model of winter wheat scab index (WSI) based on interactions between spectral information and meteorological factors. The results showed that: 1) Correlations between the daily average temperature (DAT) and daily average relative humidity (DAH) at different time scales and WSI were significant. 2) There were positive linear correlations between winter wheat biomass, leaf area index (LAI), leaf chlorophyll content (LCC) and WSI. 3) NDVI (normalized difference vegetation index), RVI (ratio vegetation index) and DVI (difference vegetation index) which had a good correlation with LAl, biomass and LCC, respectively, and could be used to replace them in modeling. 4) The estimated values of the model were consistent with the measured values (RMSE $=5.3 \%$, estimation accuracy $=$ 90.46\%). Estimation results showed that the model could efficiently estimate WS in Yangtze-Huaihe river region.
\end{abstract}

Keywords: Winter wheat scab, Spectral information; meteorological factor, Remote sensing, Yangtze-Huaihe river region

\section{Introduction}

Remote sensing takes advantages of fast and accurate in monitoring growth and disease of crop at large scale, and has been considered to be a feasible and timely method of monitoring after development of last decades (Mustafa et al. 2014; Shi et al. 2017). In early 20th century, Marek and Adam (2014) had conducted research on crop disease identification by airborne remote sensing. Tolers et al. (1981) investigated stripe rust of winter wheat by infrared aerial photography. Research from Lorenzen and Jensen (1989) indicated that barley powdery mildew severity was strongly correlated with nearinfrared band (NIR). Riedell and Blackmer (1999) studied spectral features of winter wheat leaves stressed by wheat aphid and wheat binary aphid. Devadas et al. (2009) identified and monitored leaf rust of winter wheat by using several vegetation indices. Zhao et al. (2004) studied spectral features of stripe rust of winter wheat

\footnotetext{
* Correspondence: jaaslwg@126.com Sciences, Nanjing 210014, China

Institute of Agricultural Information, Jiangsu Academy of Agricultural and established a stress index to monitor the disease. Liu et al. (2004) utilized data from airborne push-broom hyper spectral imager to monitor the degree of damage and area of winter wheat stripe rust. The research of Cai et al. (2005) showed that stress index of stripe rust of winter wheat was in good relation with canopy spectral reflectance at $930 \mathrm{~nm}$.

Prior research has shown a significant variation in the visible and near-infrared band spectral features after the crops were infected. These features have offered the theoretical basis for spectral detection of crop diseases. Moreover, healthy and infected winter wheat plants showed significant difference on spectral reflectance, and some disease index showed good quantitative relationship with canopy spectral reflectance. The research of Jiang et al. (2007) indicated stripe rust of winter wheat could be identified 12 days before its burst. Wang et al. (2008) used fluorescence spectrum to efficiently detect the severity of stripe rust of winter wheat. Forecast of winter wheat disease and conducting scientific 
prevention is critical to promote winter wheat yield and reduce economic loss.

Yangtze-Huaihe river region is one of the most important winter wheat production areas in China. Due to the monsoon climate, the spatial and temporal distribution of precipitation is not in balance and the light and temperature are variable during winter wheat growing season (Liu et al. 2017). The specific climate makes winter wheat susceptible to infection by wheat scab (WS) if the plant density is excessive. Severe WS always get a fast burst in a large area, which is hard to monitor timely.

Most researches have focused on monitoring leaf rust and powdery mildew of winter wheat from spectrum (Huang 2009; Cao et al. 2013; Ma et al. 2016; Zhang et al. 2017). However, research on monitoring WS by remote sensing is still limited. Therefore, the present study selected meteorological, agronomic and spectral factors which were strongly related to WS, to established the remote sensing estimation model of WSI. The purpose of the present study was to efficiently estimate WS, and offer information acquisition method for the prevention of winter wheat disease in Yangtze-Huaihe river region.

\section{Materials and methods}

\section{Experiment site establishment}

Experimental sites of remote sensing monitoring for WS were set up in Donghai, Lianshui, Taixing and Dafeng in 2012 and 2014. The location of 90 sampling sites were recorded via GPS. In detail, there were 10 sites for both Donghai and Lianshui, 12 sites for Taixing and 13 sites for Dafeng in each year (Fig. 1). The distance between each two sites was $2-3 \mathrm{~km}$. The sampling sites were selected in winter wheat growing patches above $300 \mathrm{~m} \times$ $300 \mathrm{~m}$, and the sampling was conducted in the middle of each patch (about $60 \mathrm{~m} \times 60 \mathrm{~m}$ ). Fertilization and irrigation of sampling sites followed the local conventional winter wheat growth management.

\section{Experimental survey and data collection}

Flower initiation stage (3-5\% of the total wheat plants are flowering in field) is the critical period for prevention of WS. Therefore, we conducted sampling at this stage. Five plots in each site (approximate $10 \mathrm{~m}$ between each plot) were selected and 10 wheat plants were sampled in each plot. GreenSeeker multi-spectrometer (Trimble, USA) was used to record spectral information (including reflectance in near-infrared and red) of winter wheat at each site. The agronomic indicators, e.g. leaf area index (LAI), biomass and leaf chlorophyll content (LCC) were obtained after sampling. The second sampling were conducted at 25 days after anthesis at the same site with the same method, and WS information was investigated simultaneously.

Meteorological data, including daily average temperature (DAT) and daily average relative humidity (DAH) were obtained from local meteorological department. Based on the meteorological data, DAT and DAH at different time scales were calculated. DAT1, DAT3, DAT5 and DAT7 represented DAT of 1, 3, 5, 7 days before (including) flower initiation, respectively and the same for DAH.

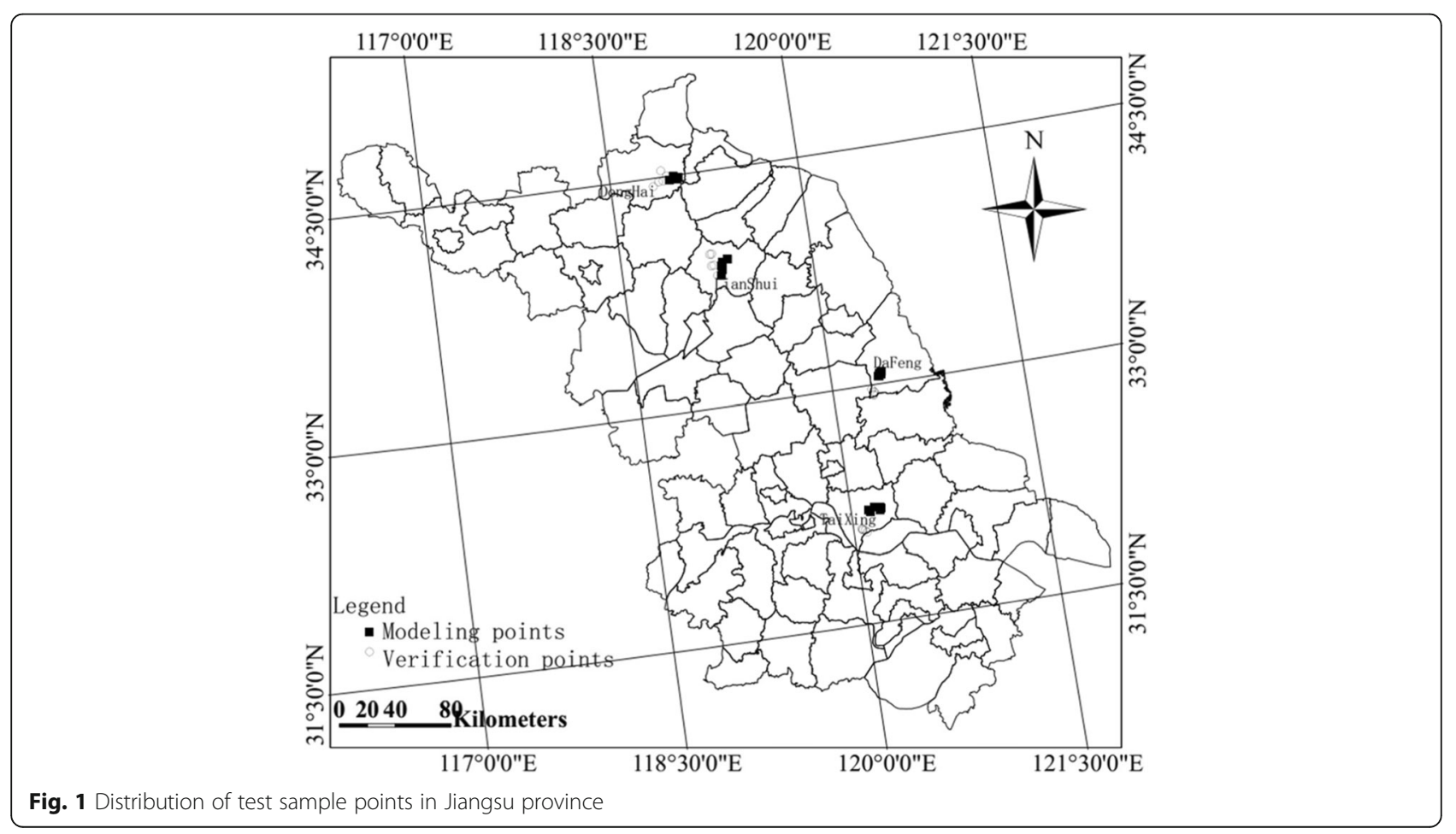


LAI of winter wheat was sampled in 5 plots for each site by SunScan (Delta-T, Britain) and the average value was used as LAI in each site. LCC of winter wheat was measured by using SPAD502 chlorophyll meter (Minolta, Japan) and average value of 10 leaves in each site was recorded. Biomass of winter wheat were obtained by sampling 10 plants in each site. The plants were initially heated for $30 \mathrm{~min}$ at $105^{\circ} \mathrm{C}$ and dried to constant weight at $75{ }^{\circ} \mathrm{C}$. The weight of the dry matter was used as biomass.

Spectral information used in the present study included NDVI (normalized difference vegetation index), RVI (ratio vegetation index) and DVI (difference vegetation index). These three indices were calculated from reflectance in near-infrared $\left(\mathrm{R}_{\text {nir }}\right)$ and red $\left(\mathrm{R}_{\text {red }}\right)$ obtained from GreenSeeker multi-spectrometer as follow.

$$
\begin{aligned}
& N D V I=\frac{R_{\text {nir }}-R_{\text {red }}}{R_{\text {nir }}+R_{\text {red }}} \\
& R V I=\frac{R_{\text {nir }}}{R_{\text {red }}} \\
& D V I=R_{\text {nir }}-R_{\text {red }}
\end{aligned}
$$

WSI was calculated by the disease degree of WS (WSD) investigated according to GB/T 15796-2011. WSD was divided into 5 classes. Class 0 to Class 4 represented that $0 \%, 0-25 \%, 25-50 \%, 50-75 \%$ and above $75 \%$ of the total spikelet was with WS, respectively. WSI was calculated as follow.

$$
\begin{aligned}
\mathrm{WSI}= & \frac{\sum_{i=0}^{4}\left(\text { plant number with } \mathrm{WSD}_{i} \times \mathrm{WSD}_{i}\right)}{\text { Total plant number } \times \operatorname{Max}(\mathrm{WSD})} \\
& \times 100 \%
\end{aligned}
$$

\section{Data utilization}

Sixty sampling points out of the total 90 ones were selected as training set, and the rest 30 ones were used as validation set. The validation set included 15 sampling sites in Donghai, Dafeng and Taixing in 2012 and 15 sampling sites in Lianshui, Dafeng and Taixing in 2014. The data used for establishment of the remote sensing estimation model of WS including WSI at 25 days after anthesis, and meteorological, agronomic and spectral data at flower initiation stage.

\section{Results and analysis}

Relationship between temperature and winter wheat scab In order to study the effect of DAT on WS. DAT with different time scales were calculated. Correlation analysis was conducted to investigate relationship between DAT and WSI. The result showed that DAT with different time scales had different relationships with WSI
(Table 1). Correlation coefficients between DAT and WSI were in the order DAT5 $>$ DAT3 $>$ DAT7 $>$ DAT10 $>$ DAT1. The highest correlation coefficient was DAT5 $(R=0.77)$, whereas the lowest was DAT1 $(R=$ 0.11). Therefore, DAT5 was the most sensitive temperature factor that influenced WS. Moreover, DAT with different time scales showed different positive correlations. The highest correlation was between DAT7 and DAT10 $(R=0.97)$. Based on the correlations, DAT with different time scales could be converted to each other.

Relationship between DAT5 and WSI is shown in Fig. 2. The results showed that DAT5 of flower initiation was in the range between 10 and $25^{\circ} \mathrm{C}$. As the increase of DAT5, WSI showed an increment. When DAT5 was between 14 and $23^{\circ} \mathrm{C}$, the severity and probability of WS all increased. The temperature between 14 and $23{ }^{\circ} \mathrm{C}$ was considered as the appropriate temperature for WS. DAT5 showed a significant positive relationship with WSI $\left(R^{2}=0.60\right)$. Thus, DAT5 was selected as a meteorological indictor to establish remote sensing estimation model of WS.

\section{Relationship between humidity and winter wheat scab}

WS is sensitive to humidity. In humid and semi-humid winter wheat growth region, especially in temperate zone like Yangtze-Huaihe river region, WS were burst frequently at large scale. Therefore, DAH was also selected to study its impact on WSI. Similar to DAT, DAH with different time scales were also calculated.

Correlation coefficients between DAH and WSI were in the order DAH5 $>$ DAH3 $>$ DAH7 $>$ DAH10 $>$ DAH1 (Table 2). The highest correlation coefficient was DAH5 $(R=0.78)$, whereas the lowest was DAH1 $(R=0.47)$. Therefore, DAH5 was the most sensitive humidity factor which impacts WS. Correlation analysis also showed that there was a positive correlation between DAH with different time scales. It is reasonable to conduct conversion of DAH with different time scales.

Relationship between DAH5 and WSI is shown in Fig. 3. The results showed that DAH5 of flower initiation was in the range between 47 and $84 \%$. As the increase of DAH5, WS showed an increment. When DAH5 was

Table 1 Correlation coefficients between DAT and WSI

\begin{tabular}{lllllll}
\hline Data & WSI & DAT1 & DAT3 & DAT5 & DAT7 & DAT10 \\
\hline WSI & 1 & & & & & \\
DAT1 & 0.11 & 1 & & & & \\
DAT3 & $0.75^{* *}$ & $0.41^{*}$ & 1 & & & \\
DAT5 & $0.77^{* *}$ & 0.25 & $0.93^{* *}$ & 1 & & \\
DAT7 & $0.72^{* *}$ & 0.18 & $0.83^{* *}$ & $0.96^{* *}$ & 1 & \\
DAT10 & $0.62^{* *}$ & 0.31 & $0.80^{* *}$ & $0.91^{* *}$ & $0.97^{* *}$ & 1 \\
\hline
\end{tabular}

*and **represent significance at $5 \%$ and $1 \%$, respectively 


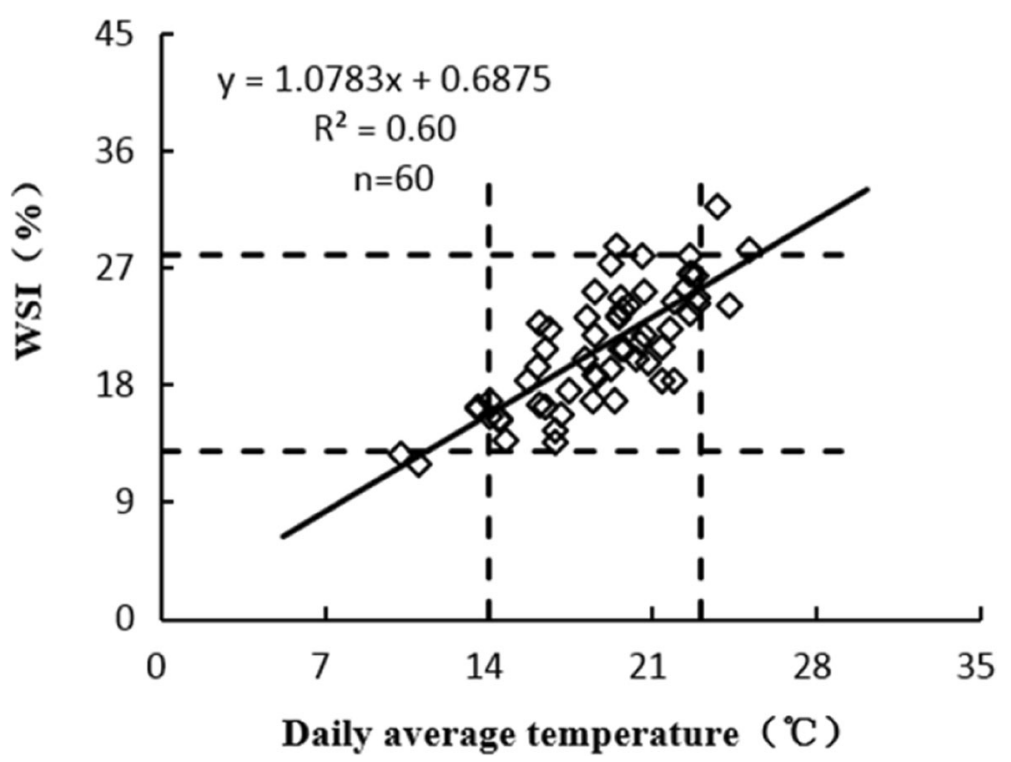

Fig. 2 The relationship between DAT5 and WSI

between 55 and $83 \%$, the severity and probability of WS all increased. The humidity between 55 and $83 \%$ was considered as the appropriate humidity for WS. DAH5 showed a significant positive relationship with WSI $\left(R^{2}=0.62\right)$. Thus, DAH5 was also selected as a meteorological indictor to build remote sensing estimation model of WS.

\section{Relationship between winter wheat growth and winter wheat scab disease}

Based on the former researches, the present study selected LAI, biomass and LCC as the agronomic indictors to investigate its relationship with WS.

Relationship between agronomic indictors and WSI showed that, most LAI of winter wheat at flower initiation stage were between 4.3 and 5.9, and significant difference was between different sampling sites (Fig. 4a). LAI is an important indicator of canopy density. If LAI was excessive, the aeration would become worse and the temperature would increase among plants, inducing a hotbed for pathogen and disease.

Table 2 Correlation coefficients between DAH and WSI

\begin{tabular}{lllllll}
\hline Data & WSI & DAH1 & DAH3 & DAH5 & DAH7 & DAH10 \\
\hline WSI & 1 & & & & & \\
DAH1 & $0.47^{*}$ & 1 & & & & \\
DAH3 & $0.75^{* *}$ & $0.79^{* *}$ & 1 & & & \\
DAH5 & $0.78^{* *}$ & $0.60^{* *}$ & $0.91^{* *}$ & 1 & & \\
DAH7 & $0.69^{* *}$ & $0.44^{*}$ & $0.77^{* *}$ & $0.91^{* *}$ & 1 & \\
DAH10 & $0.63^{* *}$ & 0.36 & $0.70^{* *}$ & $0.83^{* *}$ & $0.93^{* *}$ & 1 \\
\hline
\end{tabular}

*and ${ }^{* *}$ represent significance at $5 \%$ and $1 \%$, respectively
Winter wheat biomass at flower initiation stage were between 8.27 and $13.33 \mathrm{t} \cdot \mathrm{hm}^{-2}$ (Fig. 4b). Biomass is an important agronomic indicator of planting density. Excessive biomass at flower initiation stage would cause a competition of soil nutrients. The competition would further induce the weak wheat plants to be susceptible to infectious disease.

Most LCC of winter wheat at flower initiation stage were between 37.2 and $62.3 \%$ (Fig. 4c). LCC is an important agronomic indicator of nutrient demand of wheat plant, which could be used to guide nitrogen fertilization. Excessive nutrient of plants was not beneficial to wheat growth by causing the nutrient competition and weak plants, which was similar to excessive LAI and biomass.

In general, LAI, biomass and LCC all showed a strong positive relationship with WSI, the correlation coefficients were in an order that biomass $>$ LAI $>$ LCC $\left(R^{2}=\right.$ $0.61,0.58$ and 0.57 , respectively). The results indicated that high plant density, canopy density and over growth might be important agronomic incentives for WS. Therefore, these three parameters were selected as agronomic indicators to build remote sensing estimation model of WS.

\section{Relationship between winter wheat growth and spectral indicator}

LAI, biomass and LCC were important agronomic indicators of winter wheat growth, which could be easily obtained in field. In order to convert and quantitatively invert different winter wheat agronomic indicators from remote sensing indicators, the present study analyzed the relationship between agronomic indicators (LAI, 


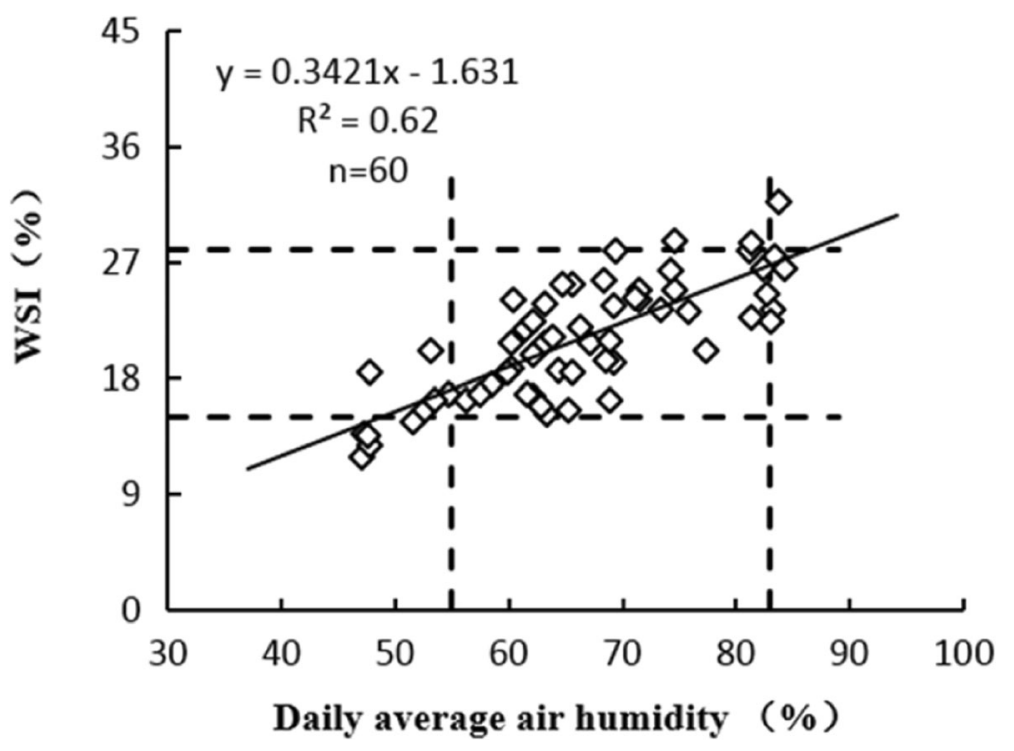

Fig. 3 The relationship between DAH5 and WSI

biomass and LCC) and remote sensing indicators $\left(\mathrm{R}_{\text {nir }}\right.$, $\mathrm{R}_{\text {red }}$, NDVI, RVI and DVI) (Table 3).

Five remote sensing indicators showed a variable relationship between each other $(R=-0.44$ to 0.97$)$. $R_{\text {nir }}$ showed a low and negative correlation with $R_{\text {red }}$, which was in accordance with the mechanism of spectral reflectance of winter wheat growth. When winter wheat grown well, the absorption of red light was enhanced, inducing reduced $R_{\text {red }}$ and enhanced $R_{\text {nir }}$, and vice versa. The internal mechanism is the scientific evidence of remote sensing monitoring of winter wheat growth. $R_{\text {nir }}$ showed a positive correlation with the three indexes $(R>0.67)$, and the highest was with DVI $(R=0.95) . \mathrm{R}_{\text {red }}$ was negative correlated with the three indexes, and the highest was with NDVI $(R=-0.94)$.

Three vegetation indexes showed a positive correlation with each other $(R>0.85)$. Based on the high correlation, the conversion of different remote sensing indicators could be achieved as a complement of single indicator. Furthermore, LAI, biomass and LCC at flower initiation stage showed positive correlation between each other $(R>0.62)$. The result could demonstrate the balance between source/sink of the photosynthesis of winter wheat.

Among these remote sensing indicators, NDVI, RVI and DVI showed strong relationship with LAI, biomass and LCC, respectively ( $R=0.85,0.85$ and 0.77$)$. Thus, the present study selected these three indexes to establish remote sensing estimation model of WS.

\section{Establishment and validation of remote sensing estimation model of WS}

Based on the strong correlation between winter wheat agronomic indicators and vegetation index of remote sensing spectrum, the present study used NDVI, RVI and DVI to replace LAI, biomass and LCC to establish the estimation model. According to the former results of

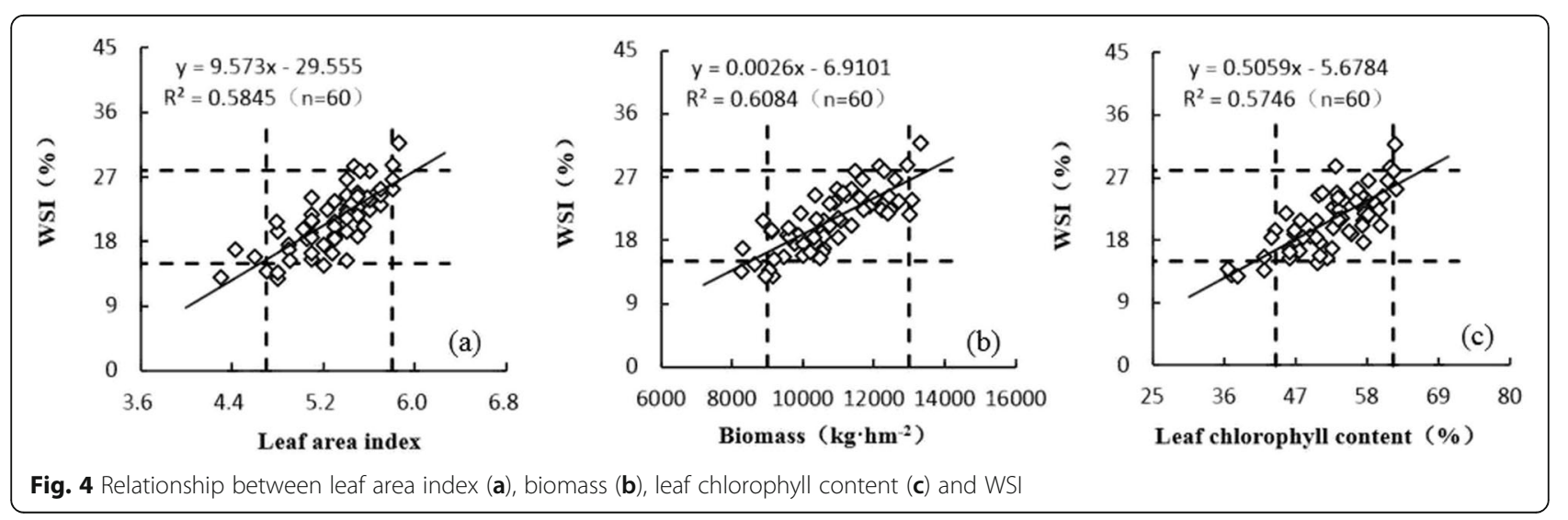


Table 3 Correlation coefficients between agronomic indicators and remote sensing spectral index of winter wheat

\begin{tabular}{lllllllll}
\hline Data & LAl & Biomass & LCC & $R_{\text {nir }}$ & $R_{\text {red }}$ & NDVI & RVI & DVI \\
\hline LAl & 1 & & & & & & & \\
Biomass & $0.77^{* *}$ & 1 & & & & & & \\
LCC & $0.63^{* *}$ & $0.63^{* *}$ & 1 & & & & & \\
R nir & $0.69^{* *}$ & $0.65^{* *}$ & $0.68^{* *}$ & 1 & & & & \\
R red & $-0.77^{* *}$ & $-0.77^{* *}$ & $-0.66^{* *}$ & $-0.44^{*}$ & 1 & & & \\
NDVI & $0.85^{* *}$ & $0.83^{* *}$ & $0.77^{* *}$ & $0.70^{* *}$ & $-0.94^{* *}$ & 1 & & \\
RVI & $0.83^{* *}$ & $0.85^{* *}$ & $0.74^{* *}$ & $0.67^{* *}$ & $-0.93^{* *}$ & $0.97^{* *}$ & 1 & \\
DVI & $0.81^{* *}$ & $0.78^{* *}$ & $0.77^{* *}$ & $0.95^{* *}$ & $-0.69^{* *}$ & $0.89^{* *}$ & $0.85^{* *}$ & 1 \\
\hline
\end{tabular}

*and **represent significance at $5 \%$ and $1 \%$, respectively

the present study, DAT5, DAH5 and the three vegetation index were selected as independent variables to calculate WSI. A remote sensing estimation model (Winter wheat Scab Remote sensing Estimating Model, WSREM) of WSI was established based on meteorological factors and spectral information, to conduct the remote sensing evaluation of WSI.

$$
\begin{aligned}
W S I_{i}= & A_{i} \times D A T 5_{i}+B_{i} \times D A H 5_{i}+C_{i} \\
& \times N D V I_{i}+D_{i} \times R V I_{i}+E_{i} \times D V I_{i}
\end{aligned}
$$

$W_{S S I}$ (Disease index of winter wheat head blight, \%) was the estimated WSI at flower initiation stage at pixel scale. DAT5 ${ }_{i}$ and $D A H 5_{i}$ were DAT5 and DAH5 at pixel scale. $N D V I_{i}, R V I_{i}$ and $D V I_{i}$ were relative index at pixel scale. $A_{i}, B_{i}, C_{i}, D_{i}$ and $E_{i}$ were model parameters.

Validation results showed that, WSI of the study region were between 11.2 and 30.1\%. Both 2012 and 2014 suffered a severe burst of WS and induced a serious wheat yield loss. The estimated WSI was agreed with measured WSI $\left(R^{2}=0.76\right)$, with a low RMSE $(5.3 \%)$ and

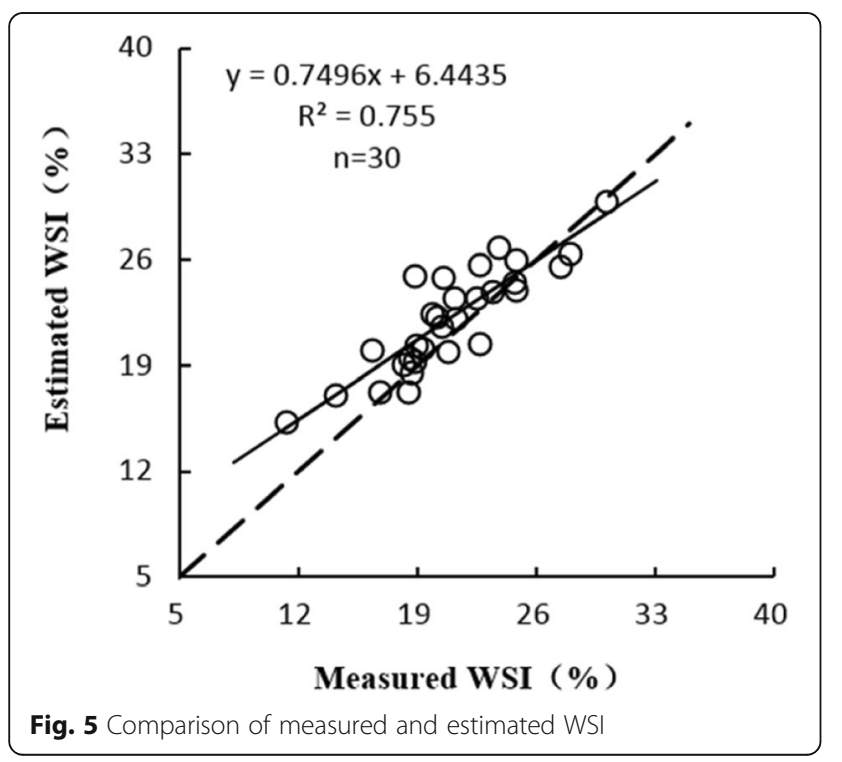

mean relative error (9.54\%) (Fig. 5). The validation result indicated that WSREM in the present study showed a good accuracy in estimating the WSI, and could be used to monitor WS at county scale.

\section{Discussion}

In traditional agriculture, winter wheat disease monitoring was conducted mainly by visual test or sampling in situ. However, these classic methods were time and energy consuming at large scale monitoring (e.g. at county scale or larger scale). Furthermore, winter wheat disease monitoring was always delayed due to the limitation of sampling area and time. Efficient disease control would not be conducted, resulting in a huge economic loss. Therefore, development of timely, large scale winter wheat disease estimation method becomes an urgent need.

Existing winter wheat disease estimation method could be divided into 3 types. The first type was based on meteorological factors (meteorological method) (Liu et al. 2008; Li et al. 2015), the second type was based on agronomic factors (agronomic method) (Si et al. 2013; Yan et al. 2017) and the third type was based on spectral information (remote sensing method) (Cai et al. 2005; Cao et al. 2013; Wang et al. 2015). These three methods had the advantages of fast, convenient and practical monitoring, but still had drawbacks such as low scientific interpretability, accuracy and feasibility. The single meteorological or agronomic method ignored the agronomic incentive or meteorological factors on winter wheat disease. The remote sensing method was based on single or multiple spectral indicators to estimate winter wheat disease. When spectral indicator of vegetation was strongly correlated with winter wheat disease indicator, the estimation results would have a good accuracy. But when the correlation was weak, the estimation result would be unreasonable. Therefore, it is necessary to take the advantages of all these three methods to improve the estimation of winter wheat disease, and to achieve a new scientific, feasible and accurate estimation of winter wheat disease.

In fact, there are many meteorological and agricultural factors of WS. However, the present study just selected the most influential factors, such as DAT and DAH as meteorological factors, LAI, biomass and LCC as agricultural factors. Other influential factors and uncertainty would also affect the accuracy of the model estimation result. Flower initiation stage is the critical growth stage for prevention of WS in Yangtze-Huaihe river region. Pesticide spraying at flower initiation stage is the traditional control strategy for WS in local area. Thus, the present study selected flower initiation stage to conduct the remote sensing estimation of WS. The estimation could be useful for the farmers on pesticide spraying management, and achieve the purpose of disease control 
and yield loss reduction. However, whether the WS estimation model of the present study could be used in other growth stages should be further investigated.

\section{Conclusions}

The WSREM model is reliable for winter wheat scab estimation at large scale. Results of the present study showed a good accuracy by comparing the measured and estimated WSI (RMSE $=5.3 \%$ and mean relative error was 9.54\%). Furthermore, this model considered both meteorological, agricultural and spectral factors, which is more reliable than models considering single factor. Based on the correlation analysis, DAT5, DAH5, NDVI, RVI and DVI were selected as the most sensitive factors on WSI and used to establish the remote sensing estimation model WSREM. The model of the present study could offer information acquisition method for WS in winter wheat production region at large scale.

\section{Acknowledgments}

This work was supported by the National Natural Science Foundation of China (No. 41571323), Key Research \& Development Plan of Jiangsu Province (BE2016730), Open Research Fund of Key Laboratory of Digital Earth Science, Institute of Remote Sensing and Digital Earth, Chinese Academy of Sciences (No.2016LDE007), and the Fund of Jiangsu Academy of Agriculture Sciences (6111647).

\section{Authors' contributions}

Conceptualization, LW; methodology, LW, CH and ZC; formal analysis, LW and LY; writing —original draft preparation, LW, CH and ZC; writing - review and editing, LW and LY. All authors read and approved the final manuscript.

\section{Funding}

This work was supported by the National Natural Science Foundation of China (No. 41571323), Key Research \& Development Plan of Jiangsu Province (BE2016730), Open Research Fund of Key Laboratory of Digital Earth Science, Institute of Remote Sensing and Digital Earth, Chinese Academy of Sciences (No.2016LDE007), and the Fund of Jiangsu Academy of Agriculture Sciences (6111647).

\section{Availability of data and materials}

Data and material are available.

\section{Competing interests}

The authors declare that they have no competing interests.

Received: 8 May 2019 Accepted: 16 January 2020

Published online: 14 February 2020

\section{References}

Cai, C. J., Ma, Z. H., Wang, H. G., Zhang, Y. P., \& Huang, W. J. (2005). Comparison research of hyperspectral properties between near-ground and high altitude of wheat stripe rust (in Chinese). Acta Phytopathologica Sinica, 1, 77-82. https://doi.org/10.3321/j.issn:0412-0914.2007.01.012.

Cao, X., Luo, Y., Zhou, Y., Duan, X., \& Cheng, D. (2013). Detection of powdery mildew in two winter wheat cultivars using canopy hyperspectral reflectance. Crop Protection, 45, 124-131.

Devadas, R., Lamb, D. W., Simpfendorfer, S., \& Backhouse, D. (2009). Evaluating ten spectral vegetation indices for identifying rust infection in individual wheat leaves. Precision Agriculture, 10, 459-470.

Huang, W. J. (2009). Remote sensing monitoring mechanism and application of crop diseases (in Chinese) (pp. 169-173). Beijing: China Agricultural Science and Technology Press.

Jiang, J. B., Chen, Y. H., \& Huang, W. J. (2007). Study on hyperspectral remote sensing retriveral models about winter wheat stripe rust severity (in Chinese) Journal-Nanjing Agricultural University, 30, 63-67.
Li, X. L., Qin, F., Zhao, L. L., Li, J. H., Ma, Z. H., \& Wang, H. G. (2015). Identification and classification of disease severity of wheat stripe rust using near infrared spectroscopy technology (in Chinese). Spectroscopy and Spectral Analysis, 35, 367-371.

Liu, L. Y., Huang, M. Y., Huang, W. J., Wang, J. H., Zhao, C. J., Zheng, L. F., \& Tong, Q. X. (2004). Monitoring stripe rust disease of winter wheat using multitemporal hyperspectral airborne data (in Chinese). Journal of Remote SensingBeijing, 8, 275-281.

Liu, W. C., Wang, J., Chen, H. L., Yu, W. D., Zhang, H., Liu, Z. Y., \& Chen, Z. F. (2008), Study on meteorological forecast model for wheat diseases and pests in Henan Province. Meteorological and Environmental Sciences, 31, 29-32.

Liu, Y., Liu, X., Shi, C., Xuan, S., \& Sun, B. (2017). Effect of biochar alleviating waterlogging stress of winter wheat in rice wheat rotation systems. Acta Pedologica Sinica, 54, 1518-1526.

Lorenzen, B., \& Jensen, A. (1989). Changes in leaf spectral properties induced in barley by cereal powdery mildew. Remote Sensing of Environment, 27, 201209.

Ma, H. Q., Huang, W. J., \& Jing, Y. S. (2016). Wheat powdery mildew forecasting in filling stage based on remote sensing and meteorological data (in Chinese). Transactions of the Chinese Society of Agricultural Engineering, 32, 165-172.

Marek, S., \& Adam, S. (2014). Image-based analysis to study plant infection with human pathogens. Computational and Structural Biotechnology Journal., 12, 1-6. https://doi.org/10.1016/j.csbj.2014.09.010.

Mustafa, M. R., James, A., Karl, S., Charles, M. R., Gerald, J. M., Fekede, W., Song, C., \& Norman, C. E. (2014). High spectral and spatial resolution hyperspectral imagery for quantifying Russian wheat aphid infestation in wheat using the constrained energy minimization classifier. Journal of applied remote sensing, 8. https://doi.org/10.1117/1.jrs. 8.083661.

Riedell, W. E., \& Blackmer, T. M. (1999). Leaf reflectance spectra of cereal aphiddamaged wheat. Crop Science, 39, 1835-1840.

Shi, Y., Huang, W. J., \& Zhou, X. F. (2017). Evaluation of wavelet spectral features in pathological detection and discrimination of yellow rust and powdery mildew in winter wheat with hyperspectral reflectance data. Journal of Applied Remote Sensing, 11, 026025.

Si, L. L., Yao, S. R., \& Yan, F. (2013). Mid-term prediction on meteorological factors for wheat powdery mildew(blumeria graminis) in Hebei Province based on fisher criterion (in Chinese). Chinese Journal of Agrometeorology, 34, 338-341.

Tolers, R. W., Smith, B. D., \& Harlan, J. C. (1981). Use of aerial color infrared photography in the study of cotton root rot. Plant Disease, 65, 24-31.

Wang, J., Jing, Y. S., Huang, W. J., Zhang, J. C., Zhao, J., Zhang, Q., \& Wang, L. (2015). Comparative research on estimating the severity of yellow rust in winter wheat. Spectroscopy and Spectral Analysis, 35, 1649-1653.

Wang, J. H., Zhao, C. J., \& Huang, W. J. (2008). Basic and application of quantitative remote sensing in agriculture (in Chinese). Beijing: Science Press.

Yan, Z. Y., Fan, J. R., Liu, W., \& Zhou, Y. L. (2017). Models of disease index estimation of wheat powdery mildew based on the concentrations of blumeria graminis f. sp. tritici conidia in the fields (in Chinese). Acta Phytopathologica Sinica, 47, 253-261.

Zhang, J. C., Pu, R. L., Yuan, L., Huang, W. J., Nie, C. W., \& Yang, G. J. (2017). Integrating remotely sensed and meteorological observations to forecast wheat powdery mildew at a regional scale. IEEE Journal of Selected Topics in Applied Earth Observations and Remote Sensing, 7, 4328-4339.

Zhao, C. J., Huang, M. Y., Huang, W. J., Liu, L. Y., \& Wang, J. H. (2004). Analysis of winter wheat stripe rust characteristic spectrum and establishing of inversion models. IEEE International Geoscience and Remote Sensing Symposium, 6, 4318-4320.

\section{Publisher's Note}

Springer Nature remains neutral with regard to jurisdictional claims in published maps and institutional affiliations. 